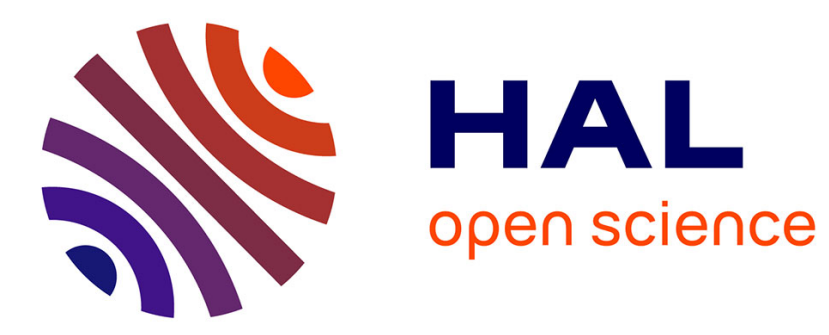

\title{
Directed Percolation Phenomena in Asynchronous Elementary Cellular Automata
}

\author{
Nazim A. Fatès
}

\section{To cite this version:}

Nazim A. Fatès. Directed Percolation Phenomena in Asynchronous Elementary Cellular Automata. Sep 2006, pp.667-675, 10.1007/11861201_77 . hal-00016420v2

\section{HAL Id: hal-00016420 \\ https://hal.science/hal-00016420v2}

Submitted on 14 Mar 2006

HAL is a multi-disciplinary open access archive for the deposit and dissemination of scientific research documents, whether they are published or not. The documents may come from teaching and research institutions in France or abroad, or from public or private research centers.
L'archive ouverte pluridisciplinaire HAL, est destinée au dépôt et à la diffusion de documents scientifiques de niveau recherche, publiés ou non, émanant des établissements d'enseignement et de recherche français ou étrangers, des laboratoires publics ou privés. 


\title{
Stochastic asynchronism may trigger phase transitions in cellular automata.
}

\author{
Nazim Fatès \\ Nazim.Fates@loria.fr
}

LORIA, University Nancy 1, France.

\begin{abstract}
Cellular automata are discrete dynamical systems that are widely used to model natural systems. Classically they are run with perfect synchrony ; i.e., the local rule is applied to each cell at each time step. A possible modification of the updating scheme consists in applying the rule with a fixed probability, called the synchrony rate. It has been shown in a previous work that varying the synchrony rate continuously could produce a discontinuity in the behaviour of the CA. This works aims at investigating the nature of this change of behaviour using intensive numerical simulations. We apply a two-step protocol to show that the phenomenon is a phase transition whose critical exponents are in good agreement with the predicted values of directed percolation.
\end{abstract}

\section{Description of the phenomenon}

This article adresses a general question raised in the modelling activity : "Does a given model keep its behaviour when it is submitted to a perturbation of its updating scheme ?" Of course, this question is too wide to be tackled in all its generality and we choose here to study it in the more narrow context of cellular automata.

In its classical paradigm, a cellular automaton consists of a collection of finite state automata arranged on a regular grid, which update their state at each time step according to a local rule. Using this formalism, we obtain discrete dynamical systems that are widely used for modelling spatially extended phenomena governed by a local rule. Such phenomena are to be found in various fields such as physics (e.g., atoms interaction in a cristal), chemistry (e.g., nonstirred reaction-diffusion), biology (e.g., virus spreading), etc. (see [1], chap. 1 for a review). The method used for assessing the validity of a model generally consists in comparing the output produced by the model to the experimental data. We claim that this step is of course necessary but that it is not sufficient : one may also need to examine to which extent the behaviour observed is due to the implicit hypotheses of the model, namely : discretization of state, regularity of the grid, perfect synchrony of the transitions.

The latter problem was at first adressed in [15] by means of simulation, the evaluation of the change in behaviour remaining qualitative. Other experimental works such as [3, 17, 16] followed, showing that the update scheme was indeed a 
key point to study. On the theoretical side, very few results have been obtained so far : e.g., the independance on the "update history" was shown undecidable in [9], existence of stationary distributions was studied in [13] and a first classification based on the convergence time was proposed in $[7]$ and extended in 87 . In the work [6], we experimentally showed that the perturbation of the updating scheme of elementary cellular automata may alter significantly the behaviour of some rules while other rules remained robust. We used one of the simplest means of introducing asynchronism in the dynamics : instead of applying the rule simultaneously to all the cells, each cell has a given probability $\alpha$, called the synchrony rate to apply the rule.

This study showed that, among other phenomena, for seven ECA, there exists a particular value of the synchrony rate $\alpha_{c}$ for which a small change of value produces an abrupt change of behaviour. It was then conjectured that this brutal variation could be explained by the existence of a phase transition, more precisely that the universality class of the phase transition was "directed percolation" (DP). We wish to emphasize that this conjecture was mainly supported by the observation of the space-time diagrams patterns produced near criticality; however, it should be noted that directed percolation was identified in other contexts such that synchronisation of two copies of cellular automata [10], probabilistic cellular automata [5] or asynchronism for the Game of Life [4].

Let a ring of $n$ cells be indexed by $\mathcal{L}=\mathbb{Z} / n \mathbb{Z}$, a configuration is word on $\{0,1\}^{\mathcal{L}}$. The density of a configuration $x$ is the ratio of cells in state 1 . An elementary cellular automaton (ECA) is described by a function $f:\{0,1\}^{3} \rightarrow$ $\{0,1\}$ called the local rule. Each ECA is indexed according to the usual notation 18 .

Using the stochastic asynchronous updating scheme, the local rule $f$ allows to define a probabilistic global rule $F$ which operates on the random variables $x^{t}$ according to $x^{0}=x$ with probability 1 and $x^{t+1}=F\left(x^{t}\right)$ such that :

$$
\forall i \in U, x_{i}^{t+1}= \begin{cases}f\left(x_{i-1}^{t}, x_{i}^{t}, x_{i+1}^{t}\right) & \text { with probability } \alpha \\ x_{i}^{t} & \text { with probability } 1-\alpha\end{cases}
$$

The rules that were experimentally detected as showing a brutal change of behaviour for a non-trivial value of $\alpha$ are ECA 6,18,26,50,58,106,146 (only "minimal representative rules" are considered). Figure 1 shows how the variation of synchrony rate affects the behaviour of three such rules. We see that two different behaviours are exhibited : either the system converges quickly to a fixed-point configuration and we say that we are in the subcritical phase, or the system evolves to a steady-state caracterised by the production of annihilatingbranching patterns, we call this steady state the supercritical phase.

At this stage, one can notice that for all the identified rules except ECA 6 , the subcritical phase is obtained for small values of $\alpha$ while for ECA 6 , it is obtained for high values of $\alpha$. It is also worth noticing that ECA 50 is a typical class-II (periodic) rule in Wolram's informal classification [18] while ECA 18 is in class III (chaotic rules). 
$\alpha=1$
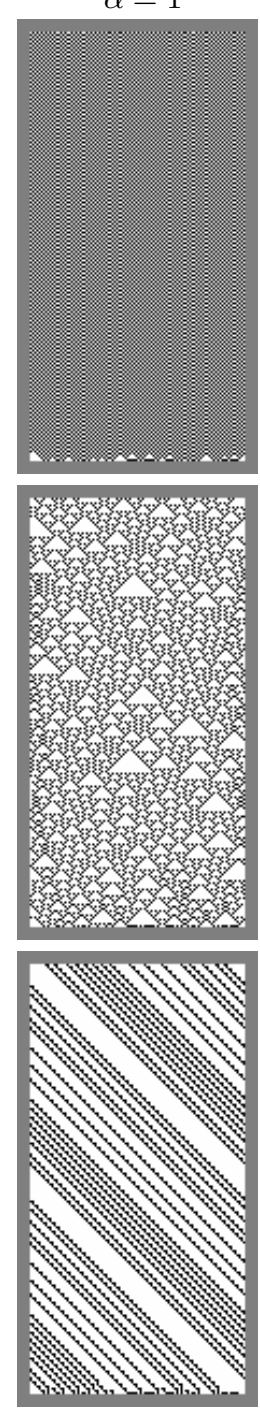

$\alpha=0.75$
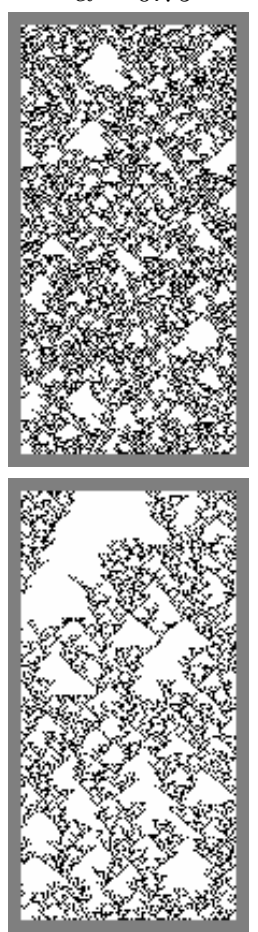

$\alpha=0.50$

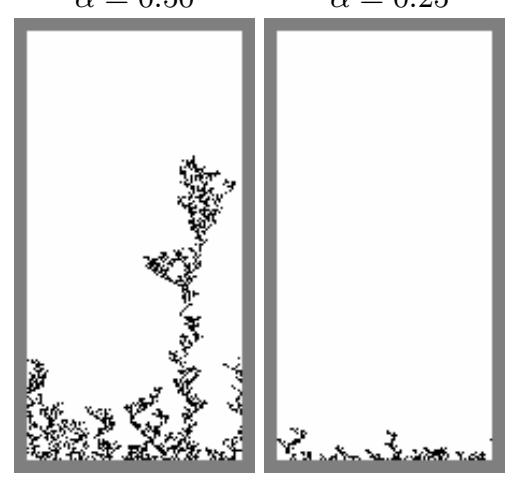

(5)
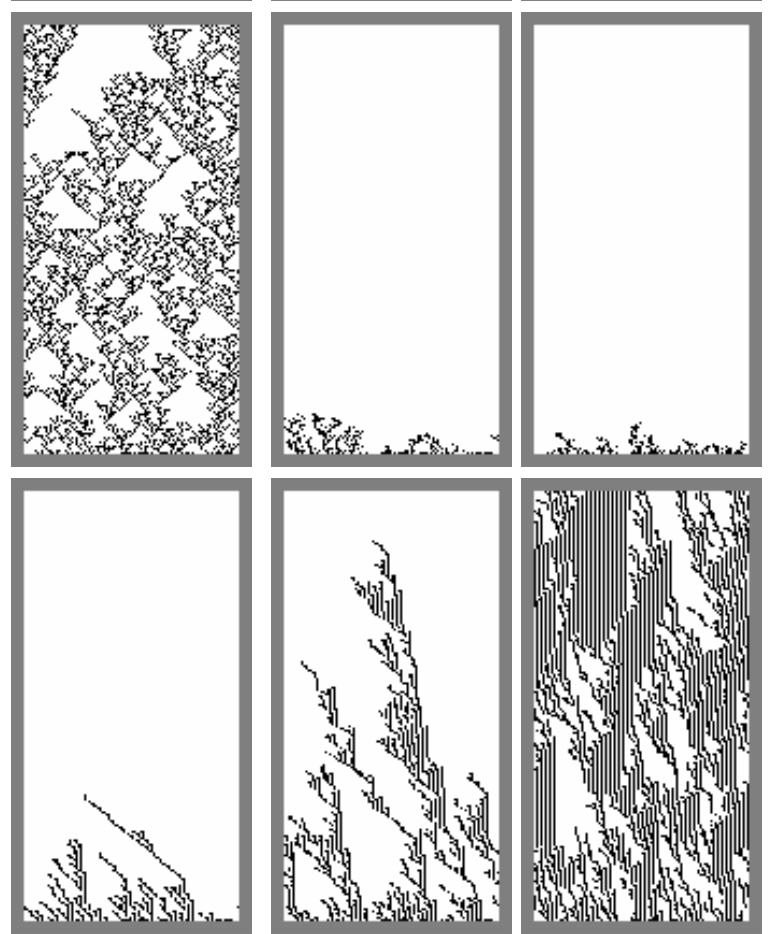

Fig. 1. The three types of phase transitions observed with ECA. The first, second and third line respectively show the evolution of ECA 50, 18 and 6. The columns show the evolutions when synchrony rate is decreased, from $\alpha=1$ (leftmost) to $\alpha=0.25$ (rightmost). In order to allow the comparison of the different asynchronous evolutions, the time factor is rescaled by a factor $1 / \alpha$ (i.e., for $\alpha=0.25$ only time steps that are multiples of 4 are displayed). 
To identify the nature of this change, we need to determine whether there exists a phase transition that can be characterised by its critical exponents or whether the observations are only due to the observation protocol. For example, one can imagine that for rule 50, the only change between subcritical and supercritical phases is a significant increase of the convergence time. In this case, the critical synchrony rate $\alpha_{c}$ would have no intrinsic signification since it would be highly dependent on the lattice size. On the other hand, if the phenomenon is indeed a phase transition that belongs to directed percolation universality class, then theory and observations [1] predict that for an infinite lattice size system, the behaviour is :

- for the subcritical phase $\alpha<\alpha_{c}$, the system converges to a frozen state $<0>=(0)_{i \in \mathcal{L}}$ with a density : $d(t)<<t^{-\delta}$ for large $t$;

- for the critical value $\alpha_{c}$, the density decreases as a power law following : $d(t) \sim t^{-\delta}$

- for the supercritical phase $\alpha>\alpha_{c}$, the system converges to a stable density $d_{\infty}(\alpha)$. The divergence from the critical state as $\alpha$ increases follows a power law: $d_{\infty}(t) \sim\left(\alpha-\alpha_{c}\right)^{\beta}$.

For the one-dimensional DP phase transition, the critical exponents are only known experimentally and are expected to take the following values : $\delta_{D P}=$ 0.1595 and $\beta_{D P}=0.2765$ (the values are given with four digits, see 12, 11] for better precision).

Naturally, these predictions only hold for infinite systems ; as simulation requires finite lattices, we are bound to introduce finite-size effects. In the following section, we explain our protocol for measuring these exponents and limiting experimental errors.

\section{Protocol}

The measure of DP-critical exponents is a delicate operation that generally requires large amount of computation time. The main difficulty resides in avoiding systematic errors when obtaining statistical data near the transition point. For example, it happened that authors were mislead by their measures and concluded that a phase transition phenomenon was not in the DP universality class [14, which was later proved wrong [10].

In order to limit the influence of systematic errors, we use the two-step protocol that was used by Grassberger [10] : we first measure the critical synchrony rate $\alpha_{\mathrm{c}}$ by varying $\alpha$ until we reach the best approximation of a power-law decay. This first experiment also allows to measure the critical exponent $\delta$. The second step consists in measuring the asymptotic density $d_{\text {as }}$ as a function of $\alpha$ and then fitting a power-law in order to calculate $\beta$. Note that teh two steps are note indpendent since the second operation uses the computed value of $\alpha_{\mathrm{c}}$.

Figure 2 shows the temporal decay of the density as $\alpha$ is varied by $10^{-3}$ steps from 0.673 to 0.677 . The curves are obtained using lattices of size $n=10000$ and by averaging the data on $Z=100$ runs of time $T=50000$. We see that as 
$\alpha$ is increased, the curve in a log-log plot transforms from a concav function to a convex function ; the best linearity is obtained for $\alpha=0.675$ and the slope measured for this value is $\delta_{146}=0.1601 \pm 0.0027$ which is close to the reference value $\delta_{D P}=0.1595$.

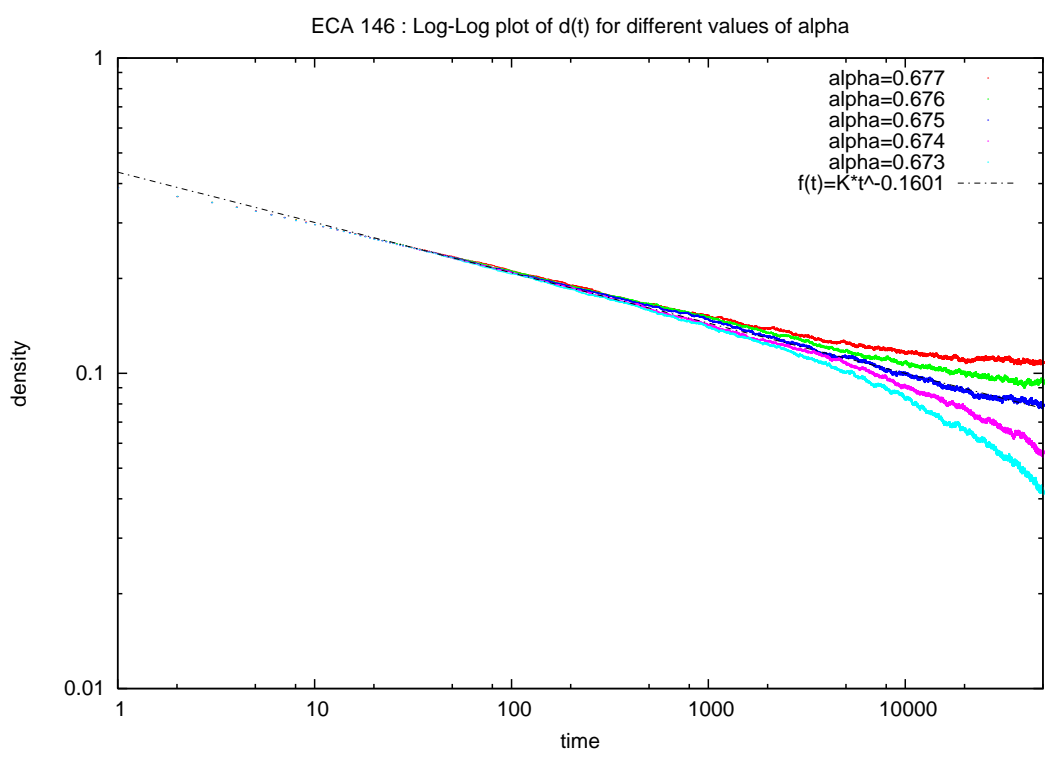

Fig. 2. ECA 146 : Determination of the critical synchrony rate $\alpha_{\mathrm{c}}$ using the time decay properties. Each plot is obtained with an average obtained on 100 runs. The curve for $\alpha=0.675$ have slope $\delta_{146}=-0.1601 \pm 0.0027$.

We iterated the measures of $d(t)$ by varying $\alpha$ with a step of $2.10^{-4}$ to bound $\alpha_{\mathrm{c}}$ between two values $\alpha_{1}$ and $\alpha_{2}$. Longer sampling times were used (up to $T=500000)$ and the convexity of the curves was determined numerically. This operation is the most time-consuming as the calculus of a single curve may require more than $10^{14}$ applications of the local rule.

Table 1 shows $\tilde{\alpha}_{\mathrm{c}}$, the best approximation of $\alpha_{\mathrm{c}}$ obtained when varying $\alpha$ and selecting the curve $d(t)$ that best fits a power-law decay. The corresponding value of the fit, $\delta\left(\tilde{\alpha}_{\mathrm{c}}\right)$, is given in the third column of the table for comparison with $\delta_{\mathrm{DP}}$. We took $t \in[2000,200000]$ as a fit interval to limit the influence of the transient time and the deviation from a power-law decay. However, it should be noted that because we have $\tilde{\alpha}_{\mathrm{c}} \neq \alpha_{\mathrm{c}}$, the temporal variation of the density will not behave as a power-law as time evolves. Thus, we cannot identify the precision on the value of $\delta$ as the precision of the fit itself. Instead, we propose to estimate the precision on $\delta$ with twe following heuristic : if we have, $\alpha_{1}<\alpha_{\mathrm{c}}<\alpha_{2}$, we use the quantity $E_{\delta}=\left|\delta\left(\alpha_{1}\right)-\delta\left(\alpha_{2}\right)\right|$. The results displayed in Table 1 show that the computed values of $\delta$ and $E_{\delta}$ are compatible with the predicted value $\delta_{\mathrm{DP}}$. 
It is interesting to notice that a variation on $\alpha$ of the order of $10^{-4}$ produces a relative variation of $10 \%$ on the value of $\delta$. This explains why $\alpha_{\mathrm{c}}$ has to be measured with high precision.

The second part of the experiments consists in measuring the critical exponent $\beta$ using the values of the asymptotic density as a function of $\alpha$. To estimate this asymptotic density, it is necessary to adjust the sampling time as $\alpha$ varies. Indeed, as $\alpha$ approaches $\alpha_{\mathrm{c}}$, the asymptotic density vanishes as $d_{\infty}(\alpha)=\left(\alpha-\alpha_{\mathrm{c}}\right)^{\beta}$, and the increase of the time needed to reach this density is thus exponential : this phenomenon, known as critical slowing down (see e.g., [11), limits the precision on the measure of the asymptotic density $d_{\infty}$.

The quantity $\Delta_{\alpha}=\alpha-\alpha_{\mathrm{c}}$ was varied according to an exponential step from 0.0032 to 0.128 . This interval is determined by the following trade-off : the computer time limits lower values of $\Delta_{\alpha}$ (critical slowing down) and for higher values of $\Delta_{\alpha}$ the system "saturates" and no longer follow a power-law. Sampling times were increased as $\Delta_{\alpha}$ was decreased and the highest sampling time $T=400000$ was used for $\Delta_{\alpha}=0.0032$.

The experiment was conducted for the seven ECA and the calculated values are shown in table 1. For each ECA, the sampling times were determined experimentally by visual estimation of the transient time (see Fig. 3). Again, the computed values of $\beta$ are in agreement with the reference value $\beta_{D P}=0.2765$.

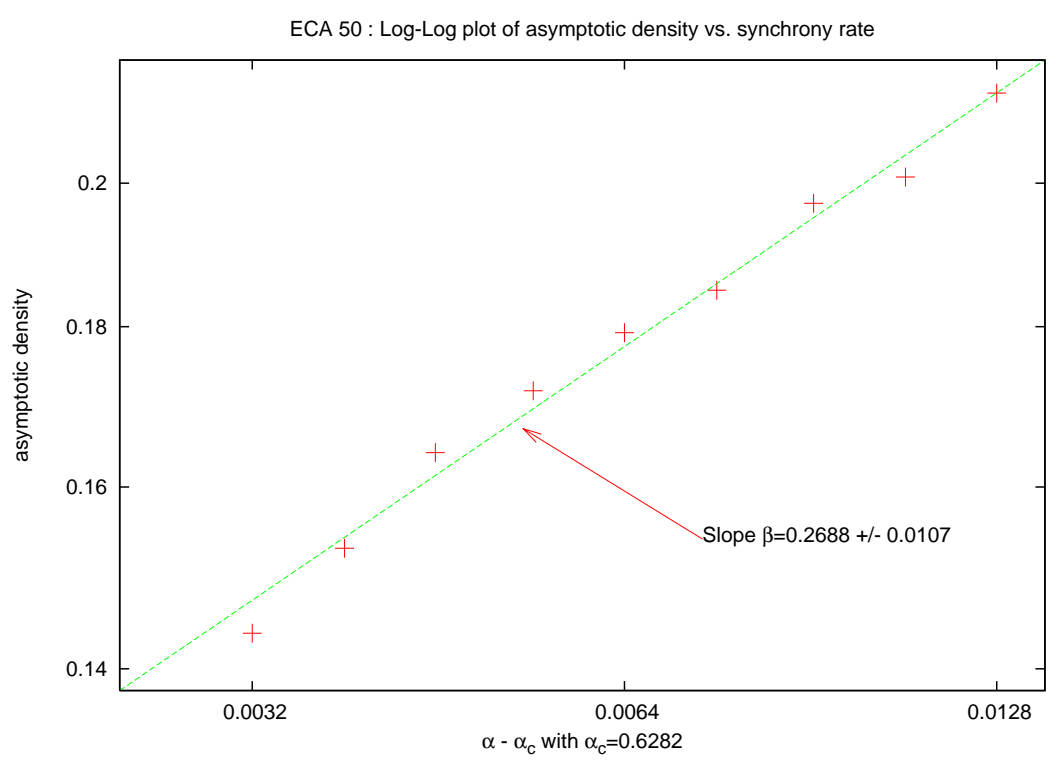

Fig. 3. ECA 50 : Determination of the critical exponent $\beta$ using the time decay properties. Each point is obtained according to using a particular sampling time (see text). The curve have slope $\beta_{50}=-0.269 \pm 0.011$. 


\begin{tabular}{|c||c|c|c|cc|}
\hline ECA & $\tilde{\alpha}_{\mathrm{c}}$ & $\delta\left(\tilde{\alpha}_{\mathrm{c}}\right)$ & $E_{\delta}$ & $\beta$ \\
\hline 6 & $0.282(4)$ & 0.158 & 0.014 & 0.265 & \pm 0.015 \\
18 & $0.713(9)$ & 0.155 & 0.028 & 0.271 & \pm 0.009 \\
26 & $0.474(8)$ & 0.164 & 0.032 & 0.264 & \pm 0.015 \\
50 & $0.628(2)$ & 0.159 & 0.024 & 0.269 & \pm 0.011 \\
58 & $0.340(0)$ & 0.162 & 0.022 & 0.270 & \pm 0.017 \\
106 & $0.814(4)$ & 0.155 & 0.023 & $0.273 \pm 0.048$ \\
146 & $0.675(0)$ & 0.163 & 0.027 & $0.259 \pm 0.021$ \\
\hline
\end{tabular}

Table 1. Critical values $\tilde{\alpha}_{\mathrm{c}}$ for the seven ECA with DP ; the last digit is uncertain. Corresponding value of $\delta$ is given with an approximation of the error on $\delta$ (see text). Critical exponent $\beta$ calculated using the given value $\tilde{\alpha}_{\mathrm{c}}$.

\section{Discussion}

The numerical simulations we presented show good evidence that the change of behaviour observed for seven asynchronous elementary cellular automata is a second order phase transition which belong to the directed percolation universality class. Improvements of precision are still required and would necessitate much more computation time but also a deeper analysis of the experimental protocol. For example, the scaling, the transients time and the fitting intervals are some parameters that should be analyzed in more detail.

The most challenging question now consists in explaining why some ECA show phase transitions while other have a smooth behaviour. The observation of the sycnhronous behaviour of the seven ECA studied indicate that there is certainly no straightforward relation with the existing classifications. Moreover, the fact that ECA 6 showed an "inversed" phase transition is surprising and this indicates that finding the relationship between the synchrony rate $\alpha$ and the probability $p$ used to describe the canonical model of directed percolation may be a difficult problem.

To conclude, let us speculate that the link showed here between statistical physics and cellular automata could benefit both domains. Indeed, on the one hand it is still an open problem to calculate analytically the values of the DP critical exponents or even to determine their nature to be rational or not. Cellular automata, by their simplicity could help making progress in the study of the DP phase transition. On the other hand, the existence of phase transitions suggests that a distributed system may change its behaviour in a totally decentralised way. For example, this mechanism could help explaining the trigger of the selforganisation phase in cellular societies [2] or allow us to design more robust decentralised network protocols.

Acknowledgments : This work was intially conducted in the LIP laboratory in Lyon (France) and then continued in the LORIA laboratory in Nancy (France). The author expresses his acknowledgments to A. Ballier and M. Morvan for their active involvement in this research, to J.-B. Rouquier for valuable suggestions. 


\section{References}

1. Andrew Adamatzky, Computing in nonlinear media and automata collectives, ISBN 075030751X, Institute of Physics Publishing, 2001.

2. Hugues Berry, Nonequilibrium phase transition in a self-activated biological network, Physical Review E (2003), no. 67, 031907.

3. Hugues Bersini and Vincent Detours, Asynchrony induces stability in cellular automata based models, Proceedings of the 4th International Workshop on the Synthesis and Simulation of Living Systems ArtificialLifeIV (Brooks, R. A, Maes, and Pattie, eds.), MIT Press, July 1994, pp. 382-387.

4. Hendrik J. Blok and Birger Bergersen, Synchronous versus asynchronous updating in the "game of life", Physical Review E 59 (1999), 3876-9.

5. Eytan Domany and Wolfgang Kinzel, Equivalence of cellular automata to ising models and directed percolation, Physical Review Letters 53 (1984), no. 4, 311314.

6. Nazim Fatès and Michel Morvan, An experimental study of robustness to asynchronism for elementary cellular automata, to appear in Complex Systems, vol 16. , arxiv:nlin.CG/0402016, 2006.

7. Nazim Fatès, Michel Morvan, Nicolas Schabanel, and Eric Thierry, Fully asynchronous behavior of double-quiescent elementary cellular automata., MFCS'05 Proceedings, vol. LNCS 3618, 2005, pp. 316-327.

8. Nazim Fatès, Nicolas Schabanel, Eric Thierry, and Damien Regnault, Asynchronous behavior of double-quiescent elementary cellular automata, to appear in LATIN'06 Proceedings, 2006.

9. P. Gács, Deterministic computations whose history is independent of the order of asynchronous updating, http://arXiv.org/abs/cs/0101026, 2003.

10. Peter Grassberger, Synchronization of coupled systems with spatiotemporal chaos, Physical Review E 59 (1999), no. 3, R2520.

11. Haye Hinrichsen, Nonequilibrium critical phenomena and phase transitions into absorbing states, Advances in Physics 49 (2000), 815-958.

12. Iwan Jensen, Low series expansions for directed percolation I. A new efficient algorithm with applications to the square lattice., Journal of Physics A 32 (1999), 5233-5249.

13. Pierre-Yves Louis, Automates cellulaires probabilistes : mesures stationnaires, mesures de gibbs associées et ergodicité, Ph.D. thesis, Université des Sciences et Technologies de Lille, September 2002.

14. Luis G. Morelli and Damian H. Zanette, Synchronization of stochastically coupled cellular automata, Physical Review E (1998), R8-R11.

15. R. L. Buvel and T.E. Ingerson, Structure in asynchronous cellular automata, Physica D 1 (1984), 59-68.

16. Andrea Roli and Franco Zambonelli, Emergence of macro spatial structures in dissipative cellular automata, Proc. of ACRI2002: Fifth International Conference on Cellular Automata for Research and Industry, Lecture Notes in Computer Science, vol. 2493, Springer, 2002, pp. 144-155.

17. Birgitt Schönfisch and André de Roos, Synchronous and asynchronous updating in cellular automata, BioSystems 51 (1999), 123-143.

18. Stephen Wolfram, Universality and complexity in cellular automata, Physica D 10 (1984), 1-35. 